\title{
Anisotropy in the extended haptic perception of longitudinal distances
}

\author{
DRAGANA BARAC-CIKOJA and M. T. TURVEY \\ University of Connecticut, Storrs, Connecticut
}

\begin{abstract}
Using horizontal rotational motions of a hand-held rod, participants in four experiments probed the aperture between two blocks separated in depth. The farther block could be either on the outer or on the inner side of the hand-rod system, defining apertures of opposite sense. The participants reported the perceived size of the in-depth intervals by adjusting, with the nonprobing hand, the lateral separation between two blocks or the egocentric distances of two blocks. Over variations in hand and geometric arrangement of stimulus and report blocks, perceived aperture size depended on the aperture's sense: Apertures with the farther edge on the outer side of the hand-rod were reported as being larger than their mirror image counterparts. The effect was not observed in judgments of a single edge distance (Experiment 5); it was configuration dependent. The sense effect would not be expected from the physical quantity accommodating perceived frontal apertures. Possible expansions of the physical model are discussed.
\end{abstract}

Recently, a number of experiments have been directed at the extended haptic perception (Burton, 1993) of frontoparallel intervals. Specifically, they have been directed at the perception of the size, $S$, of the aperture between two occluded objects at a distance, $D$, lying in a plane perpendicular to the body's sagittal plane. In these experiments, perception was achieved by horizontal probing with a hand-held rod under the condition of a fixed axis of exploratory rotation (e.g., at the wrist) located on the bisector of the angle that the aperture of a given $S$ at a given $D$ forms with respect to the axis (Barac-Cikoja \& Turvey, $1991,1993,1995)$. For a given $S$ and $D$ and a given limb segment (e.g., hand, forearm) plus probe, mechanical analysis reveals a collective parameter $\lambda_{e}$ that is invariant over the torques, impulses, and motions associated with striking the inner surfaces of the objects forming an aperture (see the Appendix for the derivation of $\lambda_{e}$ ):

$$
\lambda_{e}=\sin \alpha\left[1-(2 d / b)+\left(\operatorname{mad} / I_{o}\right)\right] .
$$

In Equation 1, $\alpha$ refers to the angular position of a limbplus-probe system at the moment of contact, relative to

This research was supported by NSF Grant SBR 94-10982 and SBR 97-09678. We thank Andrew Paladino, Steve Garett, and Mikyong Sim for assistance in conducting the experiments, Claudia Carello for Figures 1 and 3, Patrick Cabe, Barbara Sweet, Jack Loomis, and two anonymous reviewers for their comments on an earlier version of this manuscript, and Peter Brancazio for his help in revising the $\lambda$ equation. D. BaracCikoja is now at Gallaudet University, MTB, 800 Florida Ave. NE, Washington, DC 20002 (e-mail: dragana.barac-cikoja@gallaudet.edu). Correspondence concerning this article should be addressed to $M$. T. Turvey, Center for the Ecological Study of Perception and Action, Department of Psychology, University of Connecticut, Storrs, CT 06269 (e-mail: turvey@uconnvm.uconn.edu).

-Accepted by previous editor, Myron L. Braunstein the bisector of the lateral separation between the edges; $a, b$, and $d$ stand for distances from the point of rotation to the center of mass of this system, to the point of contact along the probe, and to the point of application of the driving force, respectively; $m$ is the mass of the system, and $I_{o}$ is the moment of inertia of the system with respect to the vertical axis of rotation. What Equation 1 identifies is a quantity available to the haptic system at the moment of contact with a gap edge that is specific to the complex fact, composed of a particular $S$ (more precisely, half- $S$ ) at a particular $D$, contacted by a probe with particular first and second moments of its mass distribution. $\lambda$, the sum of two $\lambda_{e} s$, therefore, captures a potential informational basis for perceived size $S^{\prime}$, as expressed in Equation 2:

$$
S^{\prime} \propto \lambda=\Sigma \lambda_{e} .
$$

Indeed, in agreement with Equation $2^{1}$ are the following observations: (1) Perception of a given $S$ decreases with $D$; (2) for the same $\alpha$, different $S$ s are perceived as different; (3) perception of a given $S$ changes with the inertial characteristics of the limb segment(s) plus probe; and (4) discrimination among a given set of $S \mathrm{~s}$ decreases at a specific rate as $D$ increases (Barac-Cikoja \& Turvey, 1991, 1993).

Although the changes in $S^{\prime}$ with translations of $S$ in depth have been accounted for by $\lambda$, there is no evidence of the generalizability of $\lambda$ across rotations of the probed apertures. The perceptual consequences of orientation changes are well documented for vision and, also, for haptic perception. Research in visual psychophysics has repeatedly demonstrated an affine compression of space along the line of sight that makes in-depth intervals look even half the size of their frontoparallel counterparts (e.g., Baird \& Biersdorf, 1967; Loomis, Da Silva, Fujita, \& Fukusima, 1992; Norman, Todd, Perotti, \& Tittle, 1996; Wagner, 1985). Interestingly, visually directed actions do not reflect such an anisotropy (see, e.g., Loomis et al., 
1992; Thomson, 1983). In the field of haptic perception, the well-known but unexplained radial-tangential effect also demonstrates that the perceived size of a physical interval arrived at by active touch depends on its orientation with respect to the observer. Specifically, a physical interval is perceived as longer when it is oriented radially from the shoulder than when it is oriented tangentially to circles centered at the shoulder (see, e.g., Day \& Wong, 1971; Marchetti \& Lederman, 1983; Reid, 1954; Wong, 1977).

The present research was aimed at expanding the database for the extended haptic perception of size to include longitudinal physical intervals-that is, the perception of the separation in depth between two objects that are at different distances from the rotation axis of the probe. The investigation should provide important constraints on the development of a more general $\lambda$-like quantity. The basic question addressed here was whether a scalar such as $\lambda$ can capture the perceived length of a spatial interval regardless of its orientation or whether a tensor of a higher order-a directed magnitude - would be needed to represent the perceptual measurement under changes in orientation. Specifically, the application of Equation 2 implies the equality of $\lambda$ values at each contact point delimiting the spatial interval. Equation 2, in other words, does not regulate how $\lambda$ values combine; only under the condition of a bilateral symmetry of the $S-D$-probe configuration is it the case that the equation's two component terms, $b$ and $\alpha$, are single valued and the equation possesses a unique solution. Clearly, orientation manipulations break the symmetry. In the case of intervals that extend at some angle to the observer's frontal plane, contact points at two different distances give rise to two different $\lambda$ values, and the effect of orientation becomes the question of the relationship between these values.

In addition, for the intervals symmetrically placed about the probe, some simplifying assumptions regarding the inertia term $I_{o}$ remained untested. In the mechanical analysis that led to Equation 1, the inertia tensor, which represents resistance of the hand-probe system to the motions in different directions, was reduced to a single moment of inertia $-I_{o}$, the one about the main axis of rotation. Moments about the other two orthogonal axes were considered irrelevant to the task, whereas the contribution of the tensor's off-diagonal terms, the products of inertia, were viewed as nonconsequential. However, research on haptic perception of the orientation of asymmetric hand-held objects (see summaries in Turvey, 1996, and Turvey \& Carello, 1995) has pointed to the role of the products of inertia. We may expect, therefore, that in the case of the asymmetric $S-D$-probe configurations, both moments and products of inertia may have discernible effects.

In the present study, we started by testing the hypothesis that, regardless of the orientation of an aperture of a given $S$, changes in $S^{\prime}$ are constrained by the overall sum of the two $\lambda$ values. To anticipate, the results of the first experiment did not support the hypothesis. Unexpectedly, an effect of the aperture sense was found: Apertures with their right edges at the further distance were perceived as larger than their mirror image counterparts. The three experiments that followed tested the reliability of the sense effect across different response configurations. Collectively, the results implicated anisotropy in distance/ location perception and a corresponding asymmetry between adductive and abductive exploratory movements. The final experiment tested the effect of this asymmetry on egocentric-distance perception and the hypothesis that $S^{\prime}$ anisometry was a consequence of the individual $D^{\prime}$ distortions. The results showed that the sense effect was configuration dependent, suggesting that haptic size perception is separable and independent from distance perception.

\section{EXPERIMENT 1}

Experiment 1 tested the hypothesis that size perception of the longitudinal intervals is uniquely determined by the sum of the $\lambda_{e}$ values, calculated for each edge independently. Thus, our starting assumption was that of the equivalence of size perception in different directions (frontoparallel and in-depth). In terms of the $\lambda$ calculations, that meant that the contributions of two $\lambda_{e}$ values were weighted equally. However, on the basis of the analogy with the anisotropies in visual perception and in some haptic tasks, we could expect that the effect of the orientation manipulation is separable from and irreducible to the distance effects that have already been demonstrated and accounted for by $\lambda$ in previous investigations. In other words, the weightings of the two $\lambda$ s differ, depending on the relative distance of the respective edges from the observer.

To reiterate, previous investigations (e.g., Barac-Cikoja \& Turvey, 1991) showed that size perception declined at farther distances: $S$ was increasingly underestimated, and also, discrimination among $S$ s diminished. These were the effects of distance on size perception that were accounted for by $\lambda$. On the basis of these findings, one can expect to see $S^{\prime}$ compressions for apertures placed farther away from an observer. However, deviations from the pattern predicted by the changes in $\lambda$ as the points of contact get increasingly separated in depth would indicate an additional effect of orientation.

\section{Method}

Participants. Eight undergraduate students (4 women and 4 men) at the University of Connecticut participated in partial fulfillment of course requirements. They had no previous experience with the task. All the participants reported being right-handed.

Materials and Apparatus. The experimental arrangement is diagrammed in Figure 1. The participant sat in front of a table (width $\times$ length $=150 \times 200 \mathrm{~cm}$ ), to the left of a curtain that extended parallel to the participant's sagittal plane throughout the length of the table and that divided the table in half. The curtain occluded the participant's right hand and the stimulus apparatus. The stimulus apparatus consisted of a pair of wooden blocks inserted in a base of support that allowed the between-block separation to be adjusted and provided stability during the exploratory striking. The position 


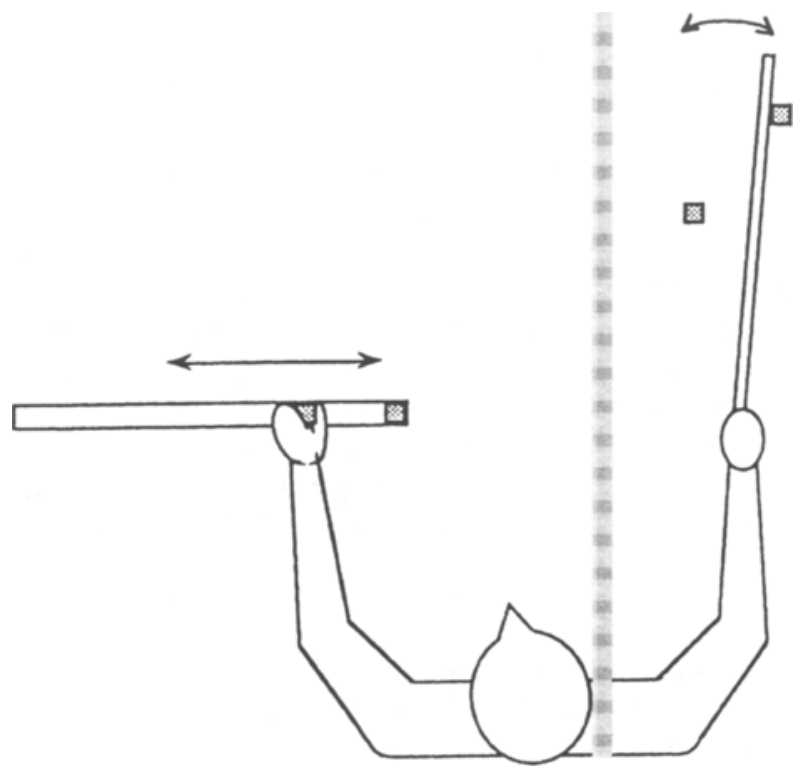

Figure 1. A schematic view of the experimental arrangement in Experiment 1. See the text for details.

of each block could change only along a course parallel to the sagittal plane, with the lateral separation between the two trails being fixed at $15 \mathrm{~cm}$. The longitudinal separation between the blocks and their distance from the participant were adjusted by one of two experimenters. The report apparatus was placed on the table to the left of the curtain, in the participant's view. The apparatus consisted of two blocks inserted in a casing. One block was glued at the right end of the casing, whereas the other could be slid to adjust the separation between the two (up to $50 \mathrm{~cm}$ ). The apparatus was arranged within a comfortable reach of the participant, to be operated with his or her left hand. Scale markings in centimeters were affixed to the back of the apparatus and were visible only to the experimenter who recorded the measurements. Each of the four blocks had a thin surface $(25 \times 7 \times 0.5 \mathrm{~cm})$ attached to its frontal side that allowed the $S$ and $D$ of the stimulus aperture to be established unambiguously and the responses to be measured precisely. The edges of the explored aperture were padded with a thin foam, to reduce substantially the sounds resulting from striking. The probe used in exploration was a $90-\mathrm{cm}$-long ( $1 \mathrm{~cm}$ in diameter) wooden rod of $38 \mathrm{~g}$ and uniform mass distribution.

Procedure. The participant was required to contact the aperture edges with the rod held in the right hand. The right forearm in midpronation was fastened to a horizontal support, with the ulnar side on the support. This provided a fixed position of the wrist throughout the experiment and allowed adduction-abduction in the horizontal plane. In its initial position, the longitudinal axis of the forearm-hand-rod was centered between the aperture edges - thus, at a 7.5-cm perpendicular distance from each. The instructions stressed that exploratory movements should be performed in the horizontal plane. There were no limitations on the manner and the rate of exploratory movements nor on the time in which to produce a measurement. The participants were instructed to adjust the size of the lateral separation between the response blocks to be the same as the size of the longitudinal distance between the stimulus blocks. It was underscored that the size of the aperture was defined by its extent in depth and that the lateral distance between the lanes of the aperture edges did not change throughout the experiment. The initial position of the adjustable response block could be at either extreme and was randomized across the trials. Four trials (with $S / D$ magnitudes different from those used in the experiment) served for a demonstration of the task. No feedback information was provided during the experiment.

Design. Longitudinal intervals of three $S \mathrm{~s}(10,15$, or $20 \mathrm{~cm})$ appeared with the farther edge at three $D \mathrm{~s}(31,64$, or $90 \mathrm{~cm}$, measured from the proximal end of the rod). The sense of the aperture was identified by the position of the farther edge, relative to the rod (left or right). For example, a right aperture of $15 \mathrm{~cm}$ at a $64-\mathrm{cm}$ distance had its right edge at $64 \mathrm{~cm}$ from the proximal end of the rod, and its left edge at a 49-cm distance. Each of the $18 \mathrm{~S} / \mathrm{D} / \mathrm{sense}$ combinations was repeated three times, and the resulting 54 trials were presented in an individually randomized order. The experimental session lasted approximately $1 \mathrm{~h}$.

\section{Results}

The values of $S^{\prime}$ averaged across trials and participants for each of the $18(S \times D \times$ sense $)$ conditions are presented in Table 1, along with the group standard deviations. A $3 \times 3 \times 2$ repeated measures analysis of variance (ANOVA) on the individual participant data (mean $S^{\prime}$ for three repetitions of each condition) showed significant effects of $S[F(2,14)=22.97, p<.0001]$ and $D[F(2,14)=$ $19.90, p<.0001]$. Consistent with the findings of previous studies (Barac-Cikoja \& Turvey, 1991, 1993, 1995), $S^{\prime}$ increased with the increase in $S$ and decreased with the increase in the overall $D$ (see Table 1). In addition, a significant effect of sense was found $[F(1,7)=8.22, p<$ $.05]$; an aperture with the right edge farther was consistently reported as being bigger than one of the same size, at the same position, but with the left edge more remote. Group means for the right- and left-directed intervals were 12.16 and $10.45 \mathrm{~cm}$, respectively. Although the individual participants differed in the magnitude of the sense effect, the overall mean right $S^{\prime}$ exceeded the mean left $S^{\prime}$ for each participant. The overall difference between two means ( $S_{\mathrm{R}}^{\prime}-S_{\mathrm{L}}^{\prime}$, in centimeters) for participants $1-8$ was $0.05,1.18,2.46,2.61,2.11,0.23,4.46$, and 0.46 , respectively. Although inspection of the means in Table 1 may suggest larger changes in $S_{\mathrm{R}}^{\prime}$ than in $S_{\mathrm{L}}^{\prime}$ with the changes in $S$, an ANOVA did not show a significant $S \times$ sense interaction $[F(2,14)=2.24, p>.1]$. However, con-

Table 1

Mean Adjusted Separation, $S^{\prime}$ (in Centimeters, With Standard Deviations), for Left- and Right-Directed Apertures as a Function of Distance, $D$, and Aperture Size, $S$, in Experiment 1

\begin{tabular}{cccccc}
\hline & & \multicolumn{3}{c}{$S^{\prime}$} \\
\cline { 3 - 4 }$D$ & & \multicolumn{2}{c}{ Left } & \multicolumn{2}{c}{ Right } \\
\cline { 3 - 6 } \cline { 5 - 6 } 31 & 10 & 14.02 & 6.27 & 15.20 & 5.05 \\
& 15 & 15.50 & 5.54 & 17.80 & 6.86 \\
& 20 & 17.89 & 8.53 & 20.45 & 6.71 \\
64 & 10 & 8.28 & 3.26 & 8.48 & 3.76 \\
& 15 & 8.49 & 2.64 & 10.20 & 2.75 \\
& 20 & 8.55 & 3.28 & 11.19 & 2.74 \\
90 & 10 & 6.81 & 2.96 & 7.69 & 4.60 \\
& 15 & 6.35 & 2.74 & 9.45 & 6.21 \\
& 20 & 8.16 & 5.15 & 8.99 & 5.20 \\
\hline
\end{tabular}




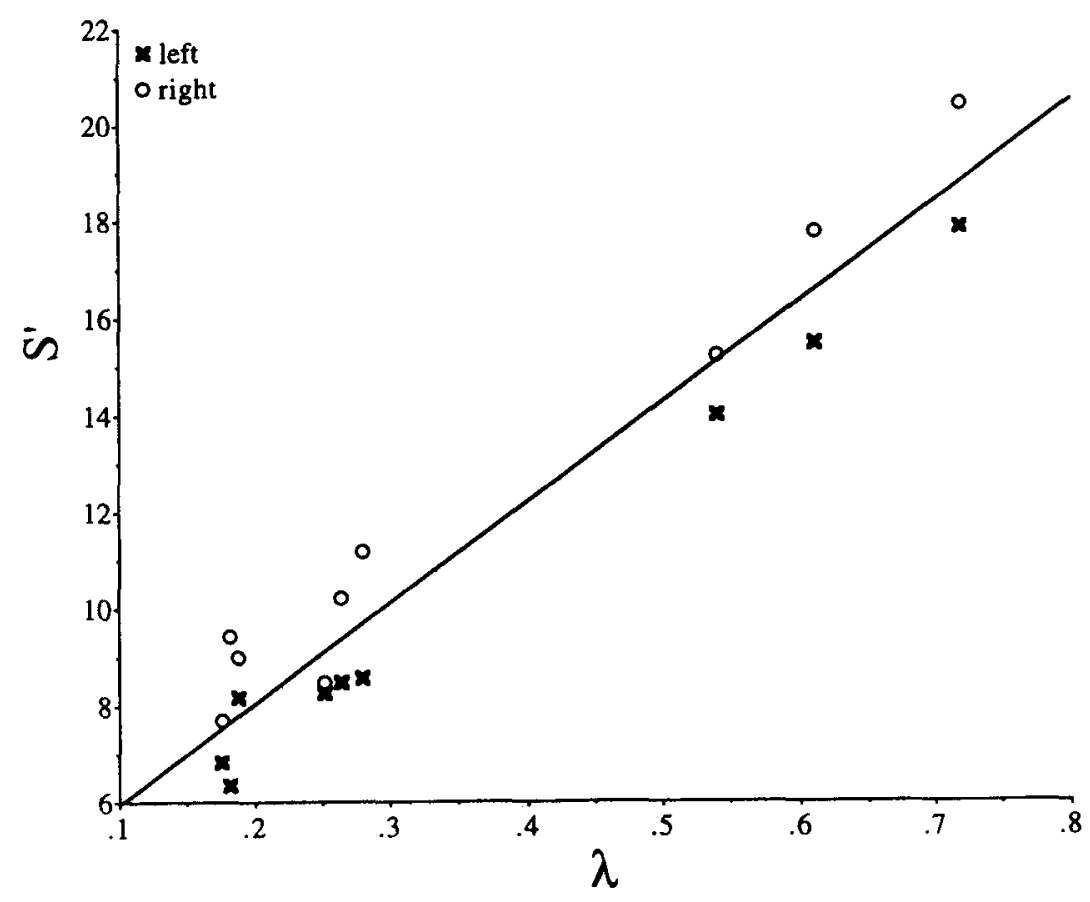

Figure 2. Results of Experiment 1 in terms of the $\lambda$ hypothesis. " $\lambda$ " refers to the sum of two equally weighted $\lambda$ values that were calculated for each edge independently.

sistent with the findings of previous studies and the $\lambda$ hypothesis, $S \times D$ was significant $[F(4,28)=3.10, p<.05]$, reflecting increased reduction in $S^{\prime}$ at a greater $D$. No other interaction reached significance.

Figure 2 shows how this pattern of results fits the expectations from the $\lambda$ hypothesis. The predictor variable is a sum of two equally weighted $\lambda_{e}$ values ${ }^{2}$ that were calculated for each edge independently, and the dependent measure is a group mean $S^{\prime}$. Filled and open circles in the graph represent left and right intervals, respectively. Clearly, the effect of sense cannot be accounted for by $\lambda$; two intervals with different senses, within the present understanding of the physical conditions, are symmetrical. For each sense, however, the general increase in $S^{\prime}$ follows the increase in $\lambda$. Regression analyses on the group means showed that $\lambda$ could account for about $94 \%$ of the total variance (individual participants' $r^{2}$ values ranged from .36 to .96). In the multiple regression analysis on individual participant means $(d f=143)$, significant contributions of both $\lambda$ (partial $F=11.98, p<.001$ ), and sense (partial $F=4.62, p<.05$ ) were found; neither $S$ nor $D$ showed significant independent effects.

\section{Discussion}

The results of the experiment on in-depth size perception are consistent with the findings of previous research on frontal size perception (Barac-Cikoja \& Turvey, 1991, 1993,1995 ), in that $S^{\prime}$ changed with both $S$ and egocentric $D$, and as such, are in agreement with the $\lambda$ hypoth- esis. Entirely unexpected, however, was the effect of interval sense. In the euclidean space of the environmental layout, which is part of the basis of $\lambda$ analysis, reflection in a line is an isometry: It does not affect the distance between the reflected points. Contrary to this understanding, the findings of Experiment 1 showed that the reflection of two edges in the line bisecting their lateral separation resulted in two different $S^{\prime}$ s: Right-oriented apertures were reproduced as being bigger than their mirror image versions.

Under the conditions of the present experimental task, two possible sources of the observed asymmetry can be identified. The source could lie in haptic perception itself and in the properties intrinsic to the haptic measurement situation. On the other hand, the demand for cross-modal matching in the size reproduction task could have introduced an independent source. In adjusting $S^{\prime}$, one might argue, the observer engaged in an imagined rigid transformation of the pair of edges that formed $S-$ a $90^{\circ}$ rotation and a translation-into another position that coincided with that of the response apparatus (cf. Carlton \& Shepard, 1990). Compared with frontoparallel intervals, then, a reproduction of the longitudinal intervals involved an additional transformation, a mental rotation of $S$, and the metric difference between $S_{\mathrm{R}}^{\prime}$ and $S_{\mathrm{L}}^{\prime}$ could have arisen from the distortions peculiar to this transformation. The following three experiments examined the conditions of size reproduction and the role that mental transformations between stimulus and response aper- 
tures might play in breaking the symmetry between right and left configurations. In doing so, they also provided a test of reliability of the findings of Experiment 1 .

That the imagined rotation has a possible role in creating the observed distortions does not preclude there being a role for factors intrinsic to the haptic perceptual system. Consider the rotational movements associated with exploration of an aperture whose edges are placed on each side of a probe. Probing the edge closer to the participant's medial plane involves the adductive hand movement, whereas probing the opposite edge involves abductive hand movements. Comparisons of adductive and abductive movements in terms of their accuracy (Poulton, 1974) and speed (Bradshaw, Bradshaw, \& Nettleton, 1988 ) have revealed that adduction was both more accurate and faster. The latter findings are usually interpreted in terms of the greater muscular tension associated with the abduction. It is conceivable that asymmetry in the resistance to movements in the two directions and, supposedly, the associated muscular effort have consequences for spatial perception as well. Striking an edge with a movement that is associated with greater effort (the outward movement of the hand-rod system) may lead to a larger perceptual estimate of the distance to the struck edge. In fact, an account of the outcome of Experiment 1 could be offered in terms of the systematic error in the egocentric distance judgments. If, given equivalent $D \mathrm{~s}$, $D^{\prime}$ for the right edge is bigger than $D^{\prime}$ for the left edge, $S$ will appear to be different for gaps of different sense: Right-directed apertures will be overestimated; leftdirected apertures will be underestimated. Patently, such a difference in $D^{\prime}$ could be attributed to the aforementioned asymmetry within the actuator.

\section{EXPERIMENT 2}

The second experiment introduced a change in the response procedure. Instead of adjusting $S^{\prime}$ in the frontal plane, the participants were required to reproduce the size of the separation in depth by adjusting the longitudinal $D$ to each edge. By having the lateral separation between the response edges fixed and equal to that of the stimulus, the new response setup allowed concurrent adjustments of the aperture size, longitudinal and oblique, of its tilt in depth, and of the egocentric distance to each edge. With this procedure, rotation as an additional spatial transformation between stimulus and response configurations was eliminated, and the conditions of reporting longitudinal and lateral $S$ were presumably equated in terms of the kind of mental transformations they might require. If rotation of $S^{\prime}$ with respect to $S$ was the sole source of metric distortions, the effect of sense observed in Experiment 1 should disappear.

The new response configuration also allowed direct examination of the accuracy of egocentric distance reports and, thereby, evaluation of the hypothesis that adjusted distance to an edge varied with the direction of the move-

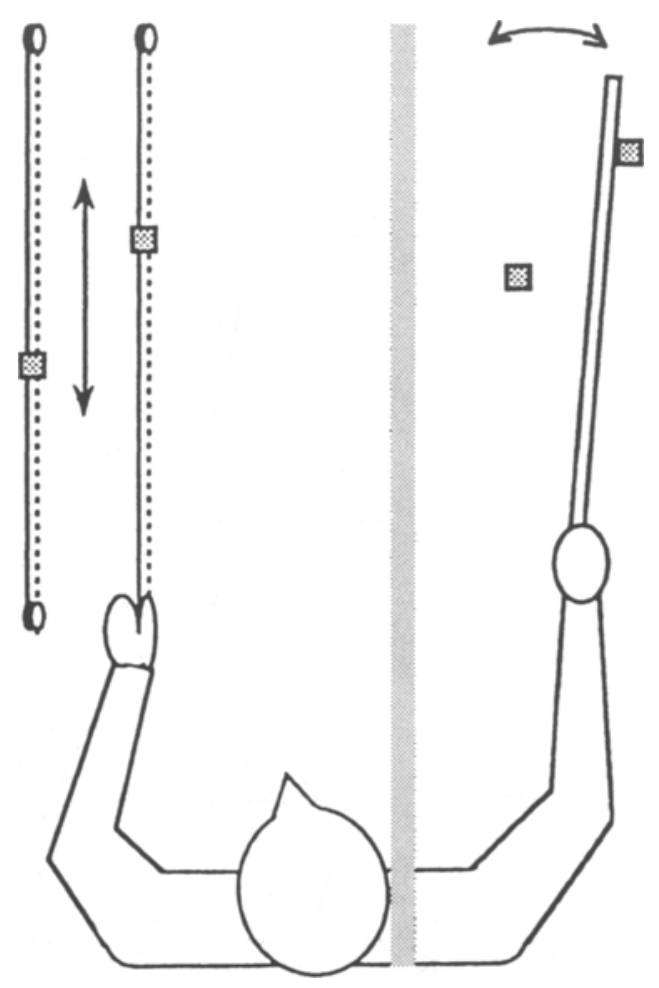

Figure 3. A depiction of the response apparatus used in Experiment 2 . See the text for details.

ment entailed in its exploration. According to the actuator asymmetry hypothesis, $D^{\prime}$ to an edge associated with abduction should exceed that associated with adduction.

\section{Method}

Participants. Seven undergraduate students (4 women and 3 men) at the University of Connecticut participated in partial fulfillment of course requirements. None of them had previous experience with the task. All the subjects reported being right-handed.

Materials and Apparatus. Figure 3 presents a diagram of the new response apparatus arrangement. Two blocks were mounted on a pair of rails that extended throughout the length of the table. Lateral separation between the rails was $15 \mathrm{~cm}$ (the same as in the stimulus apparatus). The participant moved each block along the rail by pulling a rope attached to it through a system of pulleys. The range of distances permitted by the apparatus was $0-200 \mathrm{~cm}$. Both devices could be operated within a comfortable reach. Scale markings (in centimeters) were affixed to the left side of each rail, visible only to the experimenter who recorded the measurements. As in the previous experiment, thin surfaces were attached to the frontal side of each block. In all other respects, the materials and experimental arrangement were the same as those in Experiment 1.

Procedure. The participant's task was to report the size of the longitudinal separation between two stimulus edges by manipulating egocentric distance to each response block. He or she was instructed to position the right block so as to be in the same frontal plane with the right stimulus edge and, conversely, to align the left block with the edge on the left side of the hand-rod. The instructions emphasized that the position of the stimulus blocks could change only along the tracks parallel to the rails of the response apparatus and similarly separated by a $15-\mathrm{cm}$ distance. The partici- 
Table 2

Mean Adjusted Distances (in Centimeters, With Standard Deviations) to Each Farther (f) and Closer (c) Edge for the Left- and Right-Directed Apertures, as a Function of the Actual Distance $(D)$ to the Corresponding Stimulus' Edges in Experiment 2

\begin{tabular}{|c|c|c|c|c|c|c|c|c|c|}
\hline \multirow{2}{*}{\multicolumn{2}{|c|}{$D$}} & \multicolumn{4}{|c|}{ Left } & \multicolumn{4}{|c|}{ Right } \\
\hline & & \multicolumn{2}{|c|}{$f$} & \multicolumn{2}{|c|}{$\mathrm{c}$} & \multicolumn{2}{|c|}{$\mathrm{c}$} & \multicolumn{2}{|c|}{$\mathrm{f}$} \\
\hline $\mathrm{f}$ & $\mathrm{c}$ & $M$ & $S D$ & $M$ & $S D$ & $M$ & $S D$ & $M$ & $S D$ \\
\hline 31 & 11 & 28.10 & 10.71 & 14.07 & 11.02 & 9.22 & 4.26 & 40.83 & 16.72 \\
\hline 31 & 16 & 23.67 & 6.96 & 15.14 & 8.30 & 10.19 & 4.42 & 39.12 & 19.37 \\
\hline 31 & 21 & 24.55 & 10.34 & 22.67 & 5.73 & 14.88 & 8.06 & 34.88 & 16.05 \\
\hline 64 & 44 & 68.45 & 23.39 & 56.55 & 12.77 & 39.50 & 10.51 & 65.38 & 16.44 \\
\hline 64 & 49 & 66.14 & 19.44 & 62.67 & 15.40 & 47.21 & 13.82 & 66.21 & 17.05 \\
\hline 64 & 54 & 61.41 & 17.63 & 55.69 & 11.65 & 54.34 & 19.23 & 66.45 & 19.79 \\
\hline 90 & 70 & 78.88 & 30.26 & 67.48 & 17.86 & 75.79 & 21.65 & 92.21 & 29.57 \\
\hline 90 & 75 & 80.81 & 25.23 & 81.21 & 24.24 & 70.02 & 21.89 & 81.81 & 23.82 \\
\hline 90 & 80 & 79.29 & 26.46 & 81.45 & 29.01 & 77.69 & 23.36 & 88.24 & 28.20 \\
\hline
\end{tabular}

pants were asked to pay attention to the aperture as a whole and, when adjusting individual edge positions, to keep striking both edges. In all other respects, the procedure was the same as that in Experiment 1 .

Design. Three repetitions of each size $(10,15$, or $20 \mathrm{~cm}) \times$ distance $(31,64$, or $90 \mathrm{~cm}) \times$ sense (right or left) combination were presented in an individually randomized order. On a given trial, the partıcipants produced one distance adjustment for each edge.

\section{Results and Discussion}

Table 2 shows adjusted distance $\left(D^{\prime}\right)$ to the individual edges, averaged across 7 participants, as a function of the respective actual distance $(D)$, the aperture sense (left or right), and the relative position of the edges (farther [f] or closer [c]). Since the sense of an aperture was defined by the side of its farther edge, the third and fifth columns correspond to the edges to the left of the hand-probe, and the fourth and sixth columns correspond to the edges at its right. An inspection of the means in Table 2 suggests that $D^{\prime}$ for the right edges exceeded $D^{\prime}$ for the left edge (comparisons between the sixth and fourth vs. the third and fifth, respectively). The results of the ANOVAs confirmed these observations.

For the analysis of the $D^{\prime}$ data, measurements related to the farther and the closer edges were separated. Analysis on the farther-edge data included six conditions: $3 \mathrm{Ds}$ $(31,64$, and $90 \mathrm{~cm}) \times 2$ sides (left and right); analysis on closer-edge data had 18 conditions: $9 \mathrm{Ds}(11,16,21,44$, $49,54,70,75$, and $80 \mathrm{~cm}) \times 2$ sides (left and right). A repeated measures ANOVA conducted on the individual participant data (means of the three repetitions of a given

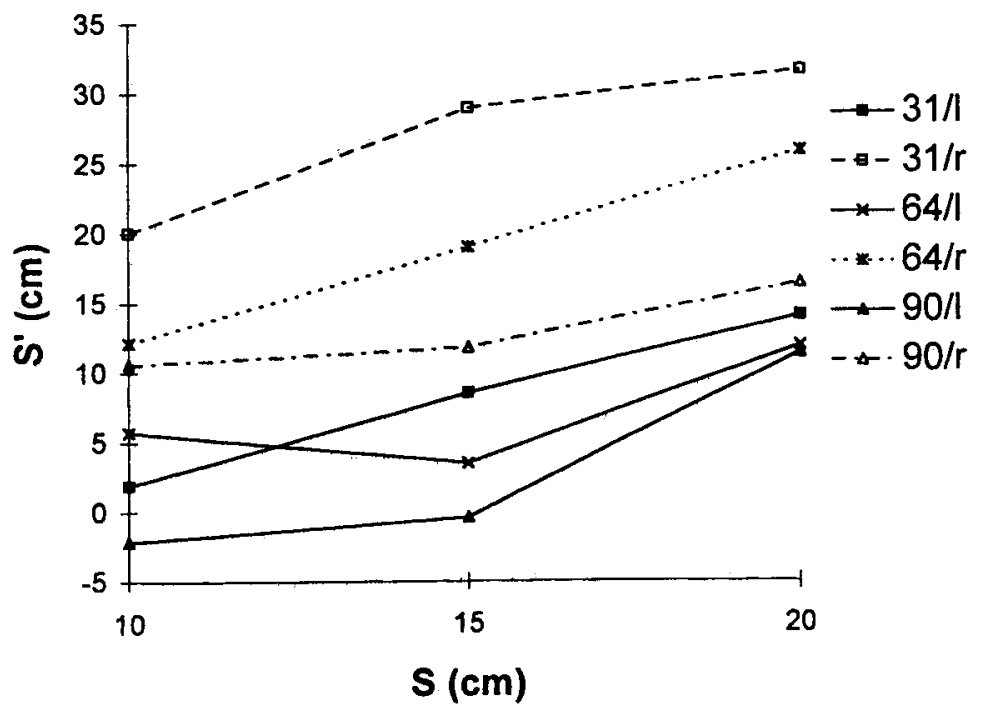

Figure 4. Adjusted longitudinal separation $\left(S^{\prime}\right)$ in Experiment 2, as a function of the actual longitudinal separation, $S$. Distance to the farther edge (31, 64,90 , in centimeters) and sense (r[ight] or l[eft]) of the stimulus aperture are curve parameters. 
condition) showed a significant effect of both variables [for the reports related to the farther edge: $D, F(2,12)=$ $47.44, p<.001$; side, $F(1,6)=14.94, p<.005$; for the reports related to the closer edge: $D, F(8,48)=53.87, p<$ .001 ; side, $F(1,6)=115.7, p<.001]$. No interaction was found to be significant in either ANOVA. In summary, $D^{\prime}$ to the left edge was consistently smaller than $D^{\prime}$ to the right edge.

Each pair of the individual $D^{\prime}$ values was converted into a measure of $S^{\prime}$ by subtracting the value corresponding to the stimulus' closer edge from that corresponding to its farther edge. This procedure yielded both positive and negative $S^{\prime}$ values (a negative sign indicating inverted aperture sense). ${ }^{3}$ Figure 4 shows $S^{\prime}$ values as a function of the size of the longitudinal separation, with $D$ and sense as curve parameters. Clearly, the size of the rightoriented apertures exceeded the size of their left-oriented counterparts. From Figure 4, it is also evident that, as in the previous experiment, $S^{\prime}$ generally increased with increases in $S$ and decreased with increases in the overall $D$. A repeated measures ANOVA on the individual participant data (means of the three repetitions of each condition) confirmed significant effects of all three variables $[S, F(2,12)=6.27, p<.02 ; D, F(2,12)=8.53, p<.005$; and sense, $F(1,6)=42.06, p<.001]$. No interaction was found significant.

In summary, the results of Experiment 2 support the attribution of the effect of aperture sense to systematic underestimation of the distance to the left edge, relative to the distance to the right edge. Contrary to the expectations from the mental rotation argument, adjustment of $S^{\prime}$ in a plane parallel to $S$ did not eliminate the effect of aperture sense. Indeed, the effect of sense grew more distinct. For each $D \times$ sense condition, a difference between the $S^{\prime}$ for corresponding right and left apertures was calculated on individual participant data from Experiments 1 and 2 and subjected to a mixed design ANOVA, with size, distance, and sense as repeated measures and experiment as a between-group factor. A significant experiment $\times$ sense effect was found $[F(1,13)=33.44, p<$ $.0001]$, confirming an increase in the difference between $S$ 's for apertures of different sense under the new conditions of reporting. Also significant was experiment $\times$ size $[F(1,13)=4.24, p<.05]$, indicating an expanded range of reproduced sizes in Experiment 2. Neither the main effect of experiment nor any other interaction was significant.

\section{EXPERIMENT 3}

In the preceding analysis of the observed metric difference between $S_{\mathrm{R}}^{\prime}$ and $S_{\mathrm{L}}^{\prime}$, we have looked into the possibility that the observer, in order to accomplish a size match, compares longitudinal distances to the respective edges of the stimulus and the response apertures. Implicit in this hypothesis is the assumption that the observer, in the cross-modal task at hand, brings to bear the Cartesian coordinate system, in which a translation along

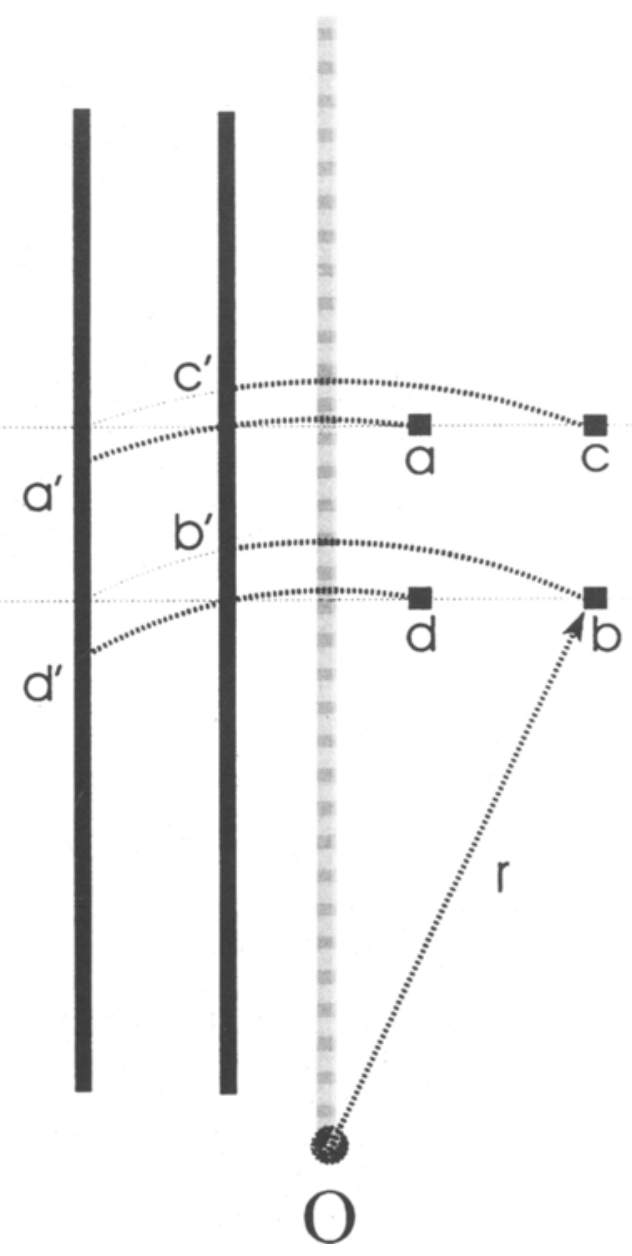

Figure 5. Schematic view of the difference between the size of the stimulus gaps ( $a b$ and $c d$ ) and the corresponding response gaps $\left(a^{\prime} b^{\prime}\right.$ and $\left.c^{\prime} d^{\prime}\right)$ when radial distances $(r)$ from the observer at $O$ to their respective right and left edges $\left(a: a^{\prime}, c: c^{\prime}\right.$, and $b: b^{\prime}$, $\left.d: d^{\prime}\right)$ are matched. Lines passing through the points $[a, d]$ and $|b, c|$, respectively, mark four points at the response side that are at equal longitudinal distances from $O$.

the orthogonal grid relates individual points on the stimulus and the response sides. Given that a translation does not change the distance between a pair of points, one could argue that the observed anisometry indicates properties that are intrinsic to the haptic system. However, the assumption of the orthogonal grid could be unwarranted, leading to a false conclusion.

Suppose that the observer, in matching $S$ and $S^{\prime}$, rather than comparing longitudinal distances, compares radial distances to the respective edges on the stimulus and the response sides. In other words, the positions of the respective edges may be compared in terms of their polar, rather than Cartesian, coordinates. Indeed, some have argued that a polar coordinate system centered at the observer is a more appropriate choice in accounting for the visual measurements of the in-depth distances than is the 
orthogonal system (e.g., Wagner, 1985). In the polar coordinate system, distance measurements are performed along the radius that extends from the pole to the point in space, and directions are specified by the angle between the radius and the polar, reference axis. Under the condition of cross-modal matching, it is plausible to assume that the pole of the common coordinate system lies in the midplane, at the crosspoint of the axis of the body's central symmetry and the plane of the stimulus and response gaps. As is demonstrated in Figure 5, points that are at the same radial distance from the pole (e.g., $a$ and $a^{\prime}$ ) lie at different longitudinal distances from the observer's frontal plane. This discrepancy is related to the difference in the angular position of (i.e., direction to) the respective points on the stimulus and the response sides imposed by the given experimental arrangement. Thus, if two right edges (on the stimulus and the response sides, respectively) are matched in their radial distance from the observer, the longitudinal distance to the response edge will be longer than the longitudinal distance to the stimulus edge. On the contrary, if the radial distances to each left edge are matched, the longitudinal distance to the response edge will be shorter than that to the stimulus edge. For the same $S$, this would render $S_{\mathrm{R}}^{\prime}$ larger than $S_{\mathrm{L}}^{\prime}$ (as is shown in Figure 5, $c^{\prime} d^{\prime}>a^{\prime} b^{\prime}$ ) and, thus, account for the pattern observed in the preceding experiments. In other words, if the individual points on the stimulus and the response sides were related by imaginal rotations in the horizontal plane (with the center of rotation being at the crosspoint of the participant's symmatry axes), rather than by frontoparallel translations, unisometry of the longitudinal distances between the points would follow.

Experiment 3 was designed to test the hypothesis that the sense effect is a result of the radial distance match between individual stimulus and response edges and, thereby, to further separate the factors related to the conditions of responding from those intrinsic to haptic spatial perception. By placing stimulus and response devices symmetrically around the medial plane and requiring that the participant produce a mirror image reproduction of the stimulus configuration, we created conditions under which angular positions, relative to the participants' midsagittal plane, as well as the radial distances of the respective edges, could be equated. This would allow the longitudinal distances to the respective edges to be equated as well and, thereby, would eliminate the difference between $S_{\mathrm{R}}^{\prime}$ and $S_{\mathrm{L}}^{\prime}$. In terms of imaginal spatial transformations, under the new conditions of responding, rotation of the individual points (i.e., each edge location) in the horizontal plane should yield size isometry. Persistence of the effect of sense, however, would provide further support for the hypothesis of spatial anisotropy in haptic perception and of actuator asymmetry as its potential basis.

\section{Method}

Participants. Eight undergraduate students ( 5 women and 3 men) at the University of Connecticut participated in partial fulfillment of course requirements. All the participants reported being righthanded. None had previous experience with the task.

Materials and Apparatus. The materials and apparatus were the same as those in Experiment 2.

Procedure and Design. The participants explored apertures with a rod held in the right hand. They were required to report the right edge position by using the left side of the response apparatus, and conversely, to report the left edge position by using the right side of the response apparatus. The instructions pointed out that the stimulus and the response devices were placed symmetrically around the participant's median plane, so that the distance from the pair of stimulus tracks to the body's median plane was the same as the visible distance to the pair of response rails. The participants were asked to produce a mirror image of the stimulus configuration by aligning the inner and the outer pair of blocks, respectively. In all other details, the procedure and the experimental design were the same as those in Experiment 2.

\section{Results and Discussion}

Table 3 shows the mean $D^{\prime}$ to each edge for the leftand right-directed apertures, respectively, as a function of $D$ to the corresponding stimulus edges. Given the request

Table 3

Mean Adjusted Distances (in Centimeters, With Standard Deviations) to

Each Farther (f) and Closer (c) Edge for the Left- and Right-Directed Apertures, as a Function of the Actual Distance $(D)$ to the Corresponding Stimulus' Edges in Experiment 3

\begin{tabular}{|c|c|c|c|c|c|c|c|c|c|}
\hline & & \multicolumn{4}{|c|}{ Left } & \multicolumn{4}{|c|}{ Right } \\
\hline \multicolumn{2}{|c|}{$D$} & \multicolumn{2}{|c|}{$\mathrm{f}$} & \multicolumn{2}{|c|}{$\mathrm{c}$} & \multicolumn{2}{|c|}{$\mathrm{c}$} & \multicolumn{2}{|c|}{$\mathrm{f}$} \\
\hline f & $\mathrm{c}$ & $M$ & $S D$ & $M$ & $S D$ & $M$ & $S D$ & $M$ & $S D$ \\
\hline 31 & 11 & 20.88 & 7.47 & 11.31 & 5.85 & 10.94 & 4.15 & 24.98 & 11.24 \\
\hline 31 & 16 & 20.60 & 10.83 & 13.98 & 11.52 & 10.17 & 4.67 & 22.98 & 15.63 \\
\hline 31 & 21 & 20.94 & 13.30 & 20.42 & 21.07 & 12.85 & 5.06 & 23.92 & 19.53 \\
\hline 64 & 44 & 53.13 & 25.98 & 48.62 & 19.80 & 35.04 & 24.21 & 49.85 & 31.07 \\
\hline 64 & 49 & 53.10 & 24.16 & 57.17 & 29.78 & 38.79 & 11.95 & 45.29 & 13.71 \\
\hline 64 & 54 & 51.19 & 22.64 & 57.23 & 28.86 & 47.69 & 22.65 & 55.90 & 25.14 \\
\hline 90 & 70 & 66.04 & 28.88 & 68.79 & 29.28 & 56.35 & 24.34 & 67.40 & 33.56 \\
\hline 90 & 75 & 65.98 & 23.28 & 72.04 & 29.42 & 61.21 & 26.15 & 66.08 & 28.29 \\
\hline 90 & 80 & 65.88 & 24.11 & 69.77 & 26.57 & 56.71 & 21.47 & 63.44 & 21.36 \\
\hline
\end{tabular}


to reproduce a mirror image of the stimulus configuration, the fourth and sixth columns correspond to the right edge on the stimulus side and to the left edge on the response side. For the third and fifth columns, the opposite is the case. An inspection of the means in Table 3 indicates the same general pattern of responses as those found in Experiment 2: $D_{\mathrm{R}}^{\prime}$ (fourth and sixth columns) exceeded $D_{\mathrm{L}}^{\prime}$ (the third and fifth columns, respectively). As in the previous experiment, individual participant $D^{\prime}$ data for the farther and for the closer edge, respectively, were subjected to separate repeated measures ANOVAs. For the reports related to the closer edge, the effects of both $D$ and side were significant $[D, F(8,56)=21.03, p<.001$; side, $F(1,7)=18.45, p<.005]$. For the reports related to the farther edge, there was a significant effect of $D[F(2,14)=$ $25.21, p<.001]$; the effect of side was not significant $[F(1,7)<1]$. No interaction was significant in either ANOVA.

In a repeated measures ANOVA on the $S^{\prime}$ values $\left(D_{\mathrm{f}}^{\prime}-D_{\mathrm{c}}^{\prime}\right)$ as the dependent measure, all three factors had significant effect $[S, F(2,14)=8.22, p<.03 ; D, F(2,14)=$ $4.24, p<.05$; and sense, $F(1,7)=7.10, p<.005$ ]. No interaction was significant. Again, $S^{\prime}$ followed changes in $S$ and decreased with increases in the overall $D$. In fact, the effect of the overall $D$, combined with the tendency to relatively overestimate distance to the right edge and underestimate distance to the left edge, resulted in an inversion of the relative position of the edges for smaller leftoriented apertures at larger $D$ s. We should point out that, although the overall pattern of findings did not change, the intertrial variability under the conditions of the present experiment rose noticeably. Upon inspection of the individual participants' responses, it seemed likely that the inversion of the left and the right edge adjustments was at times accidental. In summary, allowing $D$ and $D^{\prime}$ to be equated in polar coordinates did not eliminate the effect of aperture sense: $S^{\prime}$ was dependent on $S$ and $D$ and continued to be dependent on sense. In fact, the higher inconsistency in responses suggests that the reflection in the midplane created greater difficulty in comparing the stimulus and the response apertures. The results of Experiment 3 , therefore, invalidate the hypothesis that, in performing the cross-modal size match, the participants matched radial distances to the respective edges - that is, that they engaged in imaginal transformations on the individual edge locations that were equivalent to rotations of each point in a plane.

\section{EXPERIMENT 4}

In Experiment 4, the role of the actuator asymmetry in the observed sense effect was examined further. The spatial arrangement was the mirror image of that used in Experiment 2 . The participants explored apertures with a rod in the left hand and manipulated the distance to each edge with the right hand. Our primary question was whether the effect of aperture sense held under the condition of reflection of the spatial arrangement in the participant's sagittal plane. If the effect of aperture sense were confirmed, the goal was to establish whether the perceptual asymmetry would show reflection so that $D$ to the left edge, the one farther from the body's median plane and on the outer side of the hand-rod system, would be overestimated, relative to $D$ to the right edge. Such a reflection of size anisotropy in the sagittal plane would lend support to the interpretation in terms of the asymmetry between adduction and abduction in the exploration of the aperture edges.

\section{Method}

Participants. Eight undergraduate students ( 5 men and 3 women) at the University of Connecticut participated in partial fulfillment of course requirements. None of them had participated in similar experiments before.

Materials and Apparatus. The materials and apparatus were the same as those in Experiment 2, except that the whole experimental arrangement was reflected in the sagittal plane, so that the stimulus apparatus was placed on the left side and the response apparatus on the right side of the curtain.

Table 4

Mean Adjusted Distances (in Centimeters, With Standard Deviations) to Each Farther (f) and Closer (c) Edge for the Left- and Right-Directed Apertures, as a Function of the Actual Distance $(D)$ to the Corresponding Stimulus' Edges in Experiment 4

\begin{tabular}{|c|c|c|c|c|c|c|c|c|c|}
\hline \multirow{2}{*}{\multicolumn{2}{|c|}{$D$}} & \multicolumn{4}{|c|}{ Left } & \multicolumn{4}{|c|}{ Right } \\
\hline & & \multicolumn{2}{|c|}{ f } & \multicolumn{2}{|c|}{$\mathrm{c}$} & \multicolumn{2}{|c|}{ c } & \multicolumn{2}{|c|}{$\mathrm{f}$} \\
\hline f & $\mathrm{c}$ & $M$ & $S D$ & $M$ & $S D$ & $M$ & $S D$ & $M$ & $S D$ \\
\hline 31 & 11 & 29.5 & 11.5 & 13.2 & 5.5 & 18.6 & 14.4 & 23.9 & 13.9 \\
\hline 31 & 16 & 30.4 & 13.0 & 13.5 & 5.3 & 17.1 & 8.0 & 25.0 & 13.7 \\
\hline 31 & 21 & 29.7 & 18.2 & 14.2 & 6.9 & 21.9 & 8.2 & 23.6 & 13.8 \\
\hline 64 & 44 & 54.9 & 25.8 & 44.2 & 23.3 & 44.4 & 18.9 & 48.0 & 19.1 \\
\hline 64 & 49 & 60.1 & 24.5 & 52.7 & 22.5 & 51.7 & 22.5 & 50.7 & 21.9 \\
\hline 64 & 54 & 60.2 & 24.7 & 52.6 & 26.7 & 51.6 & 19.4 & 51.8 & 24.5 \\
\hline 90 & 70 & 64.5 & 25.5 & 55.8 & 23.3 & 62.0 & 24.0 & 61.2 & 24.4 \\
\hline 90 & 75 & 70.8 & 26.1 & 64.2 & 28.6 & 70.3 & 28.4 & 68.2 & 31.7 \\
\hline 90 & 80 & 71.9 & 26.9 & 69.7 & 29.3 & 66.4 & 22.5 & 64.2 & 25.0 \\
\hline
\end{tabular}




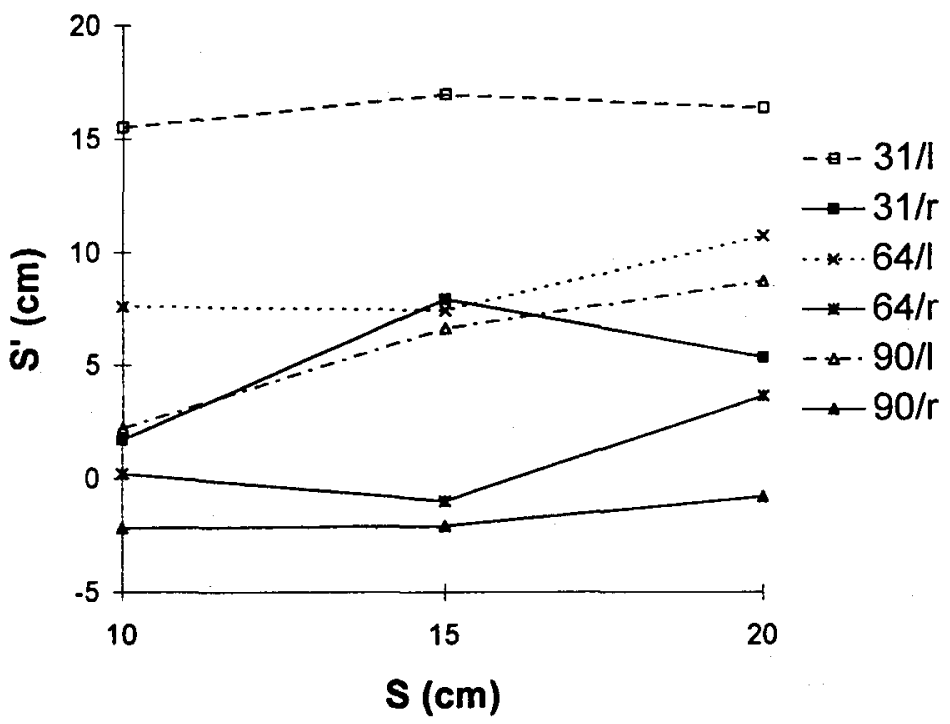

Figure 6. Adjusted longitudinal separation $\left(S^{\prime}\right)$ in Experiment 4 , as a function of the actual longitudinal separation, $S$. Distance to the farther edge (31, 64,90 , in centimeters) and sense (rlight| or l[eft|) of the stimulus aperture are curve parameters.

Procedure and Design. The participants were required to explore apertures with a rod held in the left hand and to use the right hand to produce reports. In all other details, the procedure and the experimental design were the same as those in Experiment 2.

\section{Results and Discussion}

Table 4 presents mean $D^{\prime}$ values for each edge of the left- and right-directed apertures, respectively, as a function of $D$ to the corresponding stimulus edges. An inspection of the means in Table 4 reveals the same general pattern of responses as those in the two previous experiments, except for the inversion of the sense effect. $D^{\prime}$ for the left edge (columns three and five) exceeded $D^{\prime}$ for the right edge (columns six and four, respectively). As in the two previous experiments, individual participant $D^{\prime}$ data (the means of the three repetitions of each condition) related to the farther and the closer edge, respectively, were analyzed separately in repeated measures ANOVAs. For the farther edge, both $D$ and side were significant $[D, F(2,14)=27.37, p<.001 ;$ side, $F(1,7)=$ $25.92, p<.005]$. For the closer edge, only $D$ was significant $[F(8,56)=27.44, p<.001]$; the effect of side did not reach significance $[F(1,7)=2.07, p>.1]$. No interaction was significant in either ANOVA.

In the ANOVA on the $S^{\prime}$ data, significant effects of all three repeated factors were found [ for $S, F(2,14)=8.50$, $p<.005$; for $D, F(2,14)=6.35, p<.01$; and for sense, $F(1,7)=30.85, p<.001]$. There was no significant interaction. As is apparent from Figure 6, left-oriented apertures were reported as being larger than their rightoriented counterparts, confirming the reflection of the perceptual anisotropy in the sagittal plane and providing further support for the actuator asymmetry hypothesis.

\section{EXPERIMENT 5}

Experiments 2-4 showed, with a remarkable conformity, that $D$ to an edge on the inner side of the hand-rod system was significantly underestimated, as compared with $D$ to the edge on the outer side. These findings corroborate the interpretation of size anisometry under the sense manipulation in terms of the egocentric distance anisometry, which, in turn, is due to the asymmetry between the adductive and the abductive hand movements. In the final experiment, this interpretation was examined. Contrary to the task in the preceding experiments, the participants probed a single edge located either to the right or to the left of the hand-rod system and reported its distance. Clearly, the absence of the second edge is irrelevant to the hypothesis of distance anisometry: $D^{\prime}$ for the edge on the right side (struck through abduction of the probe) should exceed $D^{\prime}$ for the edge on the left side (struck through adduction of the probe).

Table 5

Mean Adjusted Distance, $D^{\prime}$ (in Centimeters, With Standard Deviations) to an Edge Located on the Left or the Right Side of the Hand-Rod as a Function of the Actual Distance $(D)$ in Experiment 5

\begin{tabular}{crrrr}
\hline & \multicolumn{3}{c}{$D^{\prime}$} \\
\cline { 2 - 5 } & \multicolumn{2}{c}{ Left } & \multicolumn{2}{c}{ Right } \\
\cline { 2 - 5 }$D$ & $M$ & $S D$ & $M$ & $S D$ \\
\hline 10 & 9.36 & 4.54 & 12.55 & 6.27 \\
30 & 21.77 & 7.91 & 25.25 & 5.19 \\
50 & 36.67 & 11.08 & 35.12 & 16.73 \\
70 & 47.17 & 18.20 & 46.92 & 13.16 \\
90 & 53.77 & 23.56 & 52.58 & 18.30 \\
\hline
\end{tabular}




\section{Method}

Participants. Eight undergraduate students $(6$ women and 2 men) at the University of Connecticut participated in partial fulfillment of course requirements. None of them had previous experience with the task. All the participants were reported being righthanded.

Materials and Apparatus. The same materials as those from the previous experiments were used. However, at a given trial, only one stimulus block was posted for exploration, either at the left or at the right side of the hand-rod system. On the response side, the apparatus was reduced to only one track, to allow manipulation of a single block.

Procedure and Design. The participants explored the position of a single block by striking its edge with a rod held in the right hand. They were instructed to adjust the position of a response block so that it lay in the same (frontal) plane with the stimulus block. The initial position of the response block (at either extreme of the track) was randomized across trials. In all other details, the procedure was the same as that in Experiment 2. The stimulus block appeared at one of five distances (measured from the rod's proximal end): $10,30,50,70$, or $90 \mathrm{~cm}$, at either the left or the right side of the hand-rod. Ten conditions ( 5 distances $\times 2$ sides) were replicated four times in an individually randomized order. The experiment lasted about $45 \mathrm{~min}$.

\section{Results and Discussion}

Table 5 shows mean $D^{\prime}$ as a function of $D$ to the stimulus edge and its side, relative to the hand-rod. Inspection of Table 5 indicates a regular increase in $D^{\prime}$ with an increase in $D$, although at a decreasing rate. There appears to be no consistent difference with respect to the side of the inspected edge.

In a repeated measures ANOVA conducted on the individual participant data, only the effect of $D$ was found to be significant $[F(4,28)=28.35, p<.001]$. Neither side $[F(1,7)=2.67, p>.1]$ nor $D \times$ side $[F(4,28)=1.70$, $p>.1]$ was significant. At the individual participant level, not a single participant showed a significant effect of side or a significant interaction. The results of the experiment, therefore, ruled out the hypothesis that the spatial anisotropy observed in the haptic perception of the in-depth intervals rested on the asymmetry between the perceived egocentric distances, radial or rectilinear, to the edges on different sides of the hand. Evidently, perception of the position of aperture edges relative to each other is not simply a derivative of their perceived individual distances from the observer-the conclusion consistent with that reached in the previous investigation of the frontoparallel apertures (Barac-Cikoja \& Turvey, 1995).

\section{GENERAL DISCUSSION}

The departure point of the present research was the need to supplement previous investigations of extended haptic perception of frontoparallel apertures by investigations of apertures in depth. The provision of data on the relation of $S^{\prime}$ to longitudinal $S$ as a function of egocentric distance $D$ would set experimental constraints on the steps to be taken in generalizing Equation 2 . We started from the assumption that the changes in size perception related to egocentric distance can all be accounted for by the physical invariance, $\lambda$, which was derived for the frontoparallel intervals. By looking into the deviations from the pattern of $S^{\prime}$ changes predicted by $\lambda$, we would be able to estimate the independent effect of orientation.

Our intention, to determine the mechanical basis for the dependency of $S^{\prime}$ on $S$ and $D$ in Experiment 1 in the same manner of experimentation that led to Equation 2 (manipulations of $b, a$, and $I_{0}$; see Barac-Cikoja \& Turvey, 1991, 1993), was thwarted, however, by the fact that $S^{\prime}$ also depended on the direction in which an aperture was pointing -the sense of $S$. Specifically, when the farther edge forming an aperture in depth was struck with the outside surface of the rod (i.e., at a point on the rod at which the vector normal is directed away from the observer's sagittal plane), $S^{\prime}$ was greater than when the farthest edge was struck with the inside surface of the rod (where the vector normal at the point of contact is directed toward the observer's sagittal plane). This unexpected asymmetry of extended haptic perception warranted checks on its reliability. In Experiment 2, the single judgment of $S$ was replaced by reproduction of the indepth position of the edges, with $S^{\prime}$ derived as the difference between them. In Experiment 3, a mirror image reproduction of stimulus aperture was required. In Experiment 4 , the probing of apertures was done with the rod in the left hand under the conditions of Experiment 2 . The outcomes of these experiments were identical with the outcome of Experiment 1: $S^{\prime}$ was greater when the vector normal at the point of contact with the farther edge was directed away from the sagittal plane. Stated differently, $S^{\prime}$ for a given $S$ defined by an aperture extending in depth was not invariant over reflection of the near and far edges about the aperture's length.

The task of the visually controlled reproduction of the haptically explored spatial interval can be considered abstractly as a (combination of) geometric transformation(s) that bring(s) two configurations, stimulus and response, into congruence (Carlton \& Shepard, 1990). Manipulation of the conditions of responding across Experiments 1-4 can, thereby, be interpreted as a requirement for various congruence mappings between $S$ and $S^{\prime}$ : combined rotation-translation (Experiment 1), translation (Experiments 2 and 4 ), and reflection (Experiment 3). Persistence of the sense-related anisometry of $S^{\prime}$ under these transformations corroborates the haptically based interpretation.

The observed anisotropy is not an isolated finding in haptic spatial perception. Although the radial-tangential effect demonstrates affine compression of haptically perceived space along the radius originating at the arm joints, there is evidence indicating a shear, as well. In the 1930s, Walter Blumenfeld conducted experiments on kinesthetic judgments of parallelism of lines in the horizontal plane (Blumenfeld, 1936). He found that lines that the observers adjusted to be parallel to the median plane diverged toward the observer. Importantly, the closer they were to the median plane, the greater the error. In fact, 
once beyond shoulder width, adjusted lines ran parallel to the median plane. The finding bears a resemblance to the sense effect of the present study. In the dynamic touch adjustments of Blumenfeld's task, reflectional symmetry of the lines with respect to the sagittal plane running through the shoulder was broken.

Clearly, the spatial anisotropy observed in Experiments 1-4 is not addressable in the mechanical terms incorporated into Equation 2 . They can only lead to spatially symmetric outcomes. However, on the basis of the known advantage of adduction, in terms of the accuracy and speed of the movement, over abduction (see, e.g., Bradshaw et al., 1988; Poulton, 1974), one may conjecture that the observed asymmetry is brought in by the intrinsic difference between the movements toward and away from the body's median. To test the possibility that the adductionabduction asymmetry affected perceived distance to individual edges and, thereby, their longitudinal separation, Experiment 5 was conducted. Counter to this hypothesis, Experiment 5 yielded no difference in $D^{\prime}$ for single edges as a function of direction of strike. More important, perhaps, Experiment 5 showed that the results of Experiments 1-4 depended nontrivially on the spatial arrangement of a farther and a nearer edge and, thereby, on a pattern of oppositely directed impulse forces. The perceptual asymmetry of Experiments 1- 4 was configuration dependent. ${ }^{4}$

Previous investigation of aperture size perception (Barac-Cikoja \& Turvey, 1995) has also indicated that perception of the size of an interval was not a derivative of the perceived positions of its constituent (end)points within some internal coordinate system. That is, $S^{\prime} \neq \Theta D^{\prime}$, where $S^{\prime}$ and $D^{\prime}$ refer to percepts of a frontal aperture's $S$ and $D$, respectively, and $\Theta$ is the angular separation of its edges, relative to the axis of rotation. Evidently, this understanding is consistent with the hypothesis that, in the cross-modal task at hand, the observer did not infer the magnitude of the in-depth intervals from the egocentric distances to their endpoints. That is, $S^{\prime} \neq D_{\mathrm{f}}^{\prime}-D_{\mathrm{c}}^{\prime}$. For our finding of spatial anisotropy, it makes no difference whether we define the task as a perceptual measurement of $S$ along the sagittal plane or along the oblique plane connecting aperture edges. In the present study, two $S \mathrm{~s}$ were covariant, and the conditions of responding in Experiments 2-4 resulted in a reproduction of the configuration of edges, relative to the observer, allowing simultaneous adjustments of either $S$. However, an important task for future investigation is to determine what constitutes perceptually relevant aspects of the configuration. Although the physical measure of a distance between two points in space - that is, of the size of the spatial intervalis a scalar, one may argue that, perceptually, the size of an interval is inseparable from its direction with respect to the observer and, thus, can only be represented by a tensor of a higher order.

The findings of the present study also argue for further understanding of the significance of the movement direction. A pertinent conclusion was reached by Bradshaw, Bradshaw, and Nettleton (1989), who examined the accuracy of the kinesthetic judgments of extent as a function of movement direction and its position with respect to the body's midline. They found the sequence of adduction and abduction within two hemispaces (ipsiand contralateral) to be more relevant to the accuracy of the judgments than was any particular movement direction or its location. Thus, movement asymmetry, although its effect via $D^{\prime}$ is clearly ruled out, needs to be considered and tested further. If it is a relation between the consequences of exploratory adduction and those of abduction, the relative difference in the force structure created by the opposing movements may still be the symmetrybreaking factor leading to the sense effect. One could think of the geometry of the force structure (and the related tissue deformations) over the exploratory cycle as an affine shear along the frontoparallel plane. As a result, haptic sagittal plane(s) would be tilted, relative to the body's median plane, breaking the reflectional symmetry of the physical configurations about their longitudinal axis. Such spatial deformation would not affect judgments of distances on the two sides of the probe, as was the case in Experiment 5, but, consistent with the results of Experiments $1-4$, would affect size judgments. To evaluate this claim, experiments would be needed in which the aperture defining $S$ does not extend symmetrically about the probe but is decentered or positioned on either side of the probe.

The observed sense bias is, perhaps, defined more generally in terms of the reflectional asymmetry of the hand-rod mass distribution about the longitudinal axis and the consequent tilt of the hand-rod's inertia ellipsoid within the spatial coordinates of the apertures. Ellipsoid of inertia is a geometric representation of the inertia tensor, which, in turn, quantifies an object's resistance to rotations about a fixed point. For a given object and a given point of rotation, the inertia tensor is invariant. Specifically, whereas the numbers that make up the object's inertia tensor change across different extrinsic coordinate systems, their relationship is determinate and is uniquely represented by the principal moments of inertia (the eigenvalues) along the intrinsic axes of the object's inertial symmetry (the eigenvectors). These special axes and values define the size of the inertia ellipsoid and its orientation in any extrinsic coordinate system (see Turvey, 1996; Turvey \& Carello, 1995, for detailed reviews). The perceptual significance of the inertia tensor and the spatial orientation of the inertia ellipsoid have been tested in a series of experiments. Of particular significance is research directed at the relation between the perception of the arm's direction and the directions of the axes of the arm's inertia ellipsoid - that is, the eigenvectors (e.g., Pagano, Garrett, \& Turvey, 1996; Pagano \& Turvey, 1995). When the arm's eigenvectors were separated from the arm's spatial coordinates by asymmetrically weighting the arm - in other words, when the arm's inertia ellipsoid 
was tilted, relative to the longitudinal axis of the armperception of the arm's pointing direction followed the attitude of the inertia ellipsoid. How could these findings be related to the present study? We did not control for, nor did we assume a discrepancy between, the axes of the arm's inertia ellipsoid and the axes of the spatial coordinate system (either Cartesian, as in Experiments 1, 2, and 4, or polar, as in Experiment 3). To the contrary, our simplifying assumption was that of a perfectly symmetric mass distribution of a hand-rod system with respect to the longitudinal axis. A simple look at a hand with a rod, however, will reveal the ineptness of such an assumption. In other words, although the stimulus configurations in the present and previous studies (BaracCikoja \& Turvey, 1991, 1993, 1995) were symmetric with respect to the longitudinal axis, they may not have been with respect to the corresponding axis of the arm's inertia ellipsoid, the eigenvector. The existing data do not allow evaluation of this more general claim. To test it, experimental exaggerations of the inertial asymmetries of the hand-plus-probe system would be needed.

In conclusion, what general strategies are available for understanding the spatial anisotropies exhibited by the haptic perceptual system? One strategy, suggested by research in vision, is to identify an underlying geometry with metric properties that rationalizes these perceptual asymmetries. Behind this strategy is the idea that threedimensional (euclidean) physical space is mapped onto another space, a haptic space, in which the perceptions of directions and distances occur. The classical example in theorizing on space perception is the proposed mapping from Euclidean physical space to a hyperbolic visual space (Luneburg, 1947, 1950). The contrasting strategy is to identify a geometry of haptic space that, similarly, accommodates the spatial anisotropies but that varies with conditions, rather than being fixed. The idea here is that, as the configuration of environmental components and observer orientation change, so does the geometry of haptic space, but in a way that is precisely determined by the configuration (see Wagner, 1985, for discussions of this idea with regard to vision). Equations 1 and 2 are in the spirit of this latter view but depart from it in an essential respect. In extended haptic perception, variations in the physical conditions of aperture size, aperture distance, contact point along the probe, rotational inertia, and mass of the probe result in systematic variations in $\lambda$. However, $\lambda$ neither defines nor composes a haptic space. It is a mechanical quantity invariant over the forces and motions of probing that is connected to the configuration of surface layout and probe by the laws of mechanics. In terms of Gibson's perception-information specificity hypothesis (Gibson, 1959, 1966, 1979/1986; see, also, Barac-Cikoja \& Turvey, 1991, 1993), $\lambda$ is information (in the sense of information about) to which perception is specific. Barac-Cikoja and Turvey $(1991,1993)$ have suggested that perception by probing cannot exceed the specifying power of $\lambda$, in continuity with Gibson's (Reed \& Jones, 1982) suggestion that "If experience is specific to excitation, and excitation to stimulation, and stimulation to the external environment, then experience will be specific to the environment within the limits of this chain of specificity" (p. 346, authors' italics). In general, departures of $S^{\prime}$ from $S$ in extended haptic perception are explainable in terms of the systematic variations in the appropriate physical quantity.

The preceding deliberations may be summarized as follows: If extended haptic perception of size in depth is specific to a physical invariant, as has been supposed, the challenge is to identify a symmetry-breaking mechanism within the physical configuration of edges and probe and the force structure manifested by the probing activity. It seems that understanding the sense effect found in the present series of experiments must await the exact determination of how the physical symmetry of the present experimental task is broken by the exchange of the nearer and the farther edges.

\section{REFERENCES}

BAIRD, J. C., \& BiERSDORF, W. R. (1967). Quantitative functions for size and distance judgments. Perception \& Psychophysics, 2, 161-166.

Barac-Cikoja, D., \& Turvey, M. T. (1991). Perceiving aperture size by striking. Journal of Experimental Psychology: Human Perception \& Performance, 17, 330-346.

Barac-CiKoJa, D., \& TuRveY, M. T. (1993). Haptically perceiving size at a distance. Journal of Experimental Psychology: General, 122, 347-370.

BaraC-Cikoja, D., \& Turvey, M. T. (1995). Does perceived size depend on perceived distance? An argument from extended haptic perception. Perception \& Psychophysics, 57, 216-224.

BLUMENFELD, W. (1936). The relationship between the optical and haptic construction of space. Acta Psychologica, 2, 125-174.

Bradshaw, J. L., Bradshaw, J. A., \& Nettleton, N. C. (1988). Movement initiation and control: Abduction, adduction and locus of limb. Neuropsychologia, 26, 701-709.

Bradshaw, J. L., Bradshaw, J. A., \& Nettleton, N. C. (1989). Direction and location of movement in kinesthetic judgment of extent. Neuropsychologia, 27, 1139-1151.

Burton, G. (1993). Non-neural extensions of haptic sensitivity. Ecological Psychology, 5, 105-124.

Carlton, E. H., \& Shepard, R. N. (1990). Psychologically simple motions as geodesic paths: I. Asymmetric objects. Journal of Mathematical Psychology, 34, 127-188.

DAY, R. H., \& WONG, T. S. (1971). Radial and tangential movement d1rections as determinants of the haptic illusion in an $\mathrm{L}$ figure. Journal of Experimental Psychology, 87, 19-22.

DEN HARTOG, J. P. (1960). Mechanics. New York: Dover. (Original work published 1948)

Gibson, J. J. (1959). Perception as a function of stimulation. In S. Koch (Ed.), Psychology: A study of science (Vol. 1, pp. 456-501). New York: McGraw-Hill.

GiBson, J. J. (1966). The senses considered as perceptual systems. Boston: Houghton Mifflin.

GiBson, J. J. (1979/1986). The ecological approach to visual perception. Boston: Houghton Mifflin.

Loomis, J. M., DA Sil va, J. A., Fujita, N., \& Fukusima, S. S. (1992). Visual space perception and visually directed action. Journal of Experimental Psychology: Human Perception \& Performance, 18, 906-921.

LuneburG, R. K. (1947). Mathematical analysis of binocular vision. Princeton, NJ: Princeton University Press.

LUNEBURG, R. K. (1950). The metric of binocular visual space. Journal of the Optical Society of America, 40, 627-642.

MarchetTI, F. M., \& Lederman, S. J. (1983). The haptic radialtangential effect: Two tests of Wong's "moments-of-inertia" hypothesis. Bulletin of the Psychonomic Society, 21, 43-46.

Norman, J. F., Todd, J. T., Perotti, V. J., \& Tittle, J. S. (1996). The visual perception of three-dimensional length. Journal of Experimental Psychology. Human Perception \& Performance, 22, 173-186. 
Pagano, C. C., Garrett, S., \& Turvey, M. T. (1996). Is limb proprioception a function of the limbs' eigenvectors? Ecological Psychology, 8, 43-69.

Pagano, C. C., \& Turvey, M. T. (1995). The inertia tensor as a basis for the perception of limb orientation. Journal of Experimental Psychology Human Perception \& Performance, 21, 1070-1087.

Poulton, E. C. (1974). Tracking skills and manual control. New York: Academic Press.

REED, E., \& JONES, R. (1982). Reasons for realism: Selected essays of J. J. Gibson. Hillsdale, NJ: Erlbaum.

REID, R. L. (1954). An illusion of movement complementary to the horizontal-vertical illusion. Quarterly Journal of Psychology, 6, 107-111.

ThOMson, J. A. (1983). Is contınuous visual monitoring necessary in visually guided locomotion? Journal of Experimental Psychology: Human Perception \& Performance, 9, 427-443.

Turvey, M. T. (1996). Dynamic touch. American Psychologist, 51. 1134-1152.

Turvey, M. T., \& Carello, C. (1995). Dynamic touch. In W. Epstein \& S. Rogers (Eds.), Handbook of perception and cognition: V. Perception of space and motion (pp. 401-490). San Diego: Academic Press.

Wagner, M. (1985). The metric of visual space. Perception \& Psychophvsics, 38, 483-495.

WONG, T. S. (1977). Dynamic properties of radial and tangential movements as determinants of the haptic horizontal-vertical illusion with an L figure. Journal of Experimental Psvchology Human Perception \& Performance, 3, 151-164.

\section{NOTES}

1 Limb segment plus probe is a self-propelled system, and this poses a challenge as to how to most appropriately represent the muscular forces that create torques and rotatıons at the joint In our original analyses, we chose to represent $F_{\mathrm{m}}$ as a force that is recruited from within the system to act on the system as an external force (Other representations are possible, leading to different formulations of the probing task; Barbara Sweet, personal communication, August 1996). In our initial formulation (Barac-Cikoja \& Turvey, 1991, 1993, 1995), an integral force $F_{\mathrm{m}}$ was assumed to act on the center of mass of the whole system (e.g., hand + rod, forearm + hand + rod, or the whole arm + rod) to actuate its rotation about the system's fixed end. As a result, our original $\lambda_{c^{\prime}}$ equation differed from the present one (see Equation 1):

$$
\lambda_{c^{\prime}}=\sin \alpha\left[1-(2 a / b)+\left(m a^{2} / l_{b}\right)\right] .
$$

Equation 3 has been shown to accommodate the data and to lead to nonobvious predictions (Barac-Cikoja \& Turvey, 1991, 1993, 1995). The present form of the $\lambda_{c}$ equation (Equation I) represents a refinement of Equation 3 by being more precise about the point within the system where the driving force is applied.

2 . In calculatıng $\lambda_{c}$, the component parametric values were approxımated as follows: $m=0.41 \mathrm{~kg}, I_{b}=106.8 \mathrm{~kg} \cdot \mathrm{cm}^{2}, a=6 \mathrm{~cm}, d=1 \mathrm{~cm}$.

3 . By measuring $S^{\prime}$ as a difference between two $D^{\prime}$ values. we are not suggesting a perceptual or cognitive derivation of a size percept from two distance/location percepts. Furthermore, the task of adjustıng the locations of the two blocks may not involve the same function as the task of adjusting a separation between the two blocks. In other words, $S^{\prime}$ obtained from the two $D^{\prime}$ values may not be the same as $S^{\prime}$ obtained from a direct match of longitudinal intervals (see below). We thank $\mathrm{J}$. Loomis for bringing this distinction to our attention.

4. In visual research, a dissociatıon between egocentric and exocentric distance perception has been indicated as well. Particularly pertinent is a finding by Loomis and associates (Loomis et al., 1992) that the anisotropy found in the perception of spatial intervals (exocentric distances) does not characterize the perception of individual locations (egocentric distances). Investigation in the haptic domain, therefore, may provide a unıque contribution to the understanding of this differentiation in the perception of spatial layout.

\section{APPENDIX}

During the time of contact with an aperture edge, a set of forces can be identified that act on the tissues of the haptic system at the point of rotation to deform and, thereby, stimulate them (see Barac-Cikoja \& Turvey, 1991, pp. 336-337, for an explication of the nature of the forces involved). One such force, $S_{O}$, accompanies a force $S$ that is applied by the edge (at the distance $b$ from the point of rotation $O$ ) to the hand-held rod during a strike. From the equations of motion under combined forces and torques, the magnitude of this impulsive force can be derived in terms of $S$ (den Hartog, 1948/1960):

$$
S_{O}=-S\left(1-m a b / I_{o}\right)
$$

where $S_{O}, S$, and $b$ are as defined above; $m$ is the mass of the hand + rod system; $a$ is the distance from the center of mass of the system to $O$; and $I_{o}$ is the moment of inertia of the system around the $y$-axis of rotation at $O$ (in this study, $O$ being in the wrist). $S$ and $S_{O}$ are forces that cause the system (i.e., the handwith-rod) to bounce.

If we suppose that the subject provides the muscular force, $F$, to arrest that motion, there is another force, $F_{O}$, acting at $O$ that accompanies $F$ :

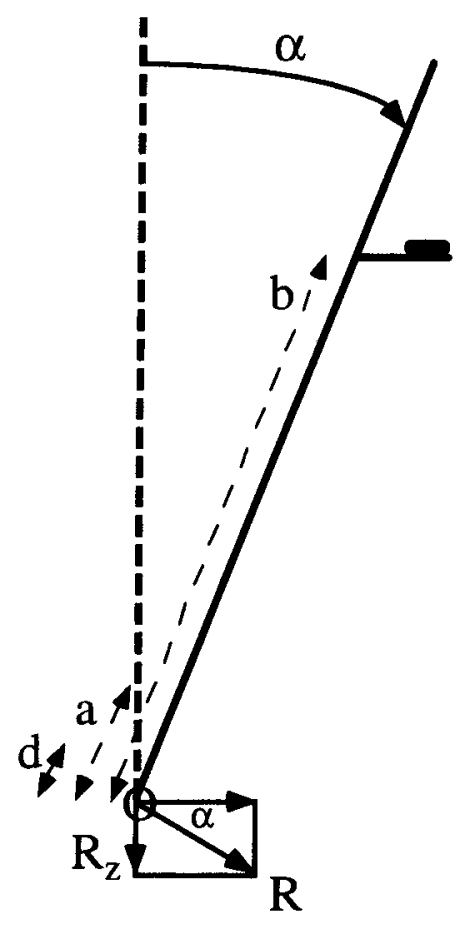

Figure A1. A diagram identifying some of the variables appearing in the derivation of $\lambda$. At the time of contact with the aperture edge, the force $S$ is applied by the edge at distance $b$ from the point of rotation at $O$, and the muscular force $F$ is applied at distance $d$ from $O$. The center of mass of the hand + probe system is at distance $a$ from $O$. The $R_{z}$ component of the resultant force $R$ acts along the longitudinal axis $z$ that bisects the lateral separation of the edges. The angular position of the system, relative to the $z$-axis, is given by $\alpha$. 


\section{APPENDIX (Continued)}

$$
F_{O}=-F\left(1-\operatorname{mad} / I_{o}\right),
$$

where $F_{O}, F, m, a$, and $I_{o}$ are as defined above and $d$ is the distance from $O$ to the point where $F$ is applied to the system. The diagram in Figure Al identifies the variables appearing in Equations A1-A2.

The effect of $S_{O}$ and $F_{O}$ on the tissues of the haptic system can be substituted by the effect of their vector sum, $R$. After $S_{O}$ is expressed in terms of $F$ :

$$
S_{O}=-F\left[\left(2 \mathrm{mad} / I_{o}\right)-(2 d / b)\right],
$$

the magnitude of this resultant force becomes

$$
R=-F\left[1-(2 d / b)+\left(\operatorname{mad} / I_{o}\right)\right] .
$$

The muscular force $F$ and the resultant force $R$ always act parallel to each other and orthogonal to the system; the negative sign indicates that they point in opposite directions. At the time of collision with the aperture edge, force $R$ acts at an angle $\alpha$ relative to the longitudinal axis--that is, the axis parallel to the forearm centered between the edges. This angle, in turn, is determined by the spatial configuration-that is, the angular position of the edge, relative to the longitudinal axis and the rotation point at $O$. Thus, the magnitude of the force component acting on the tissues along the longitudinal axis, $R_{z}=R \sin \alpha$, reflects the spatial configuration of the edges, relative to the point of rotation of the exploratory system, and potentially specifies that configuration.
Significantly, whereas the magnitude of $F$ and, thereby, of $R$ can clearly vary independent of the system involved in exploration (i.e., the arm segments and the tool), as well as of the spatial configuration, the part of Equation A4 in the brackets identifies a quantity unique to these factors. More precisely, this quantity is specific to the mass distribution of the system ( $m, a$, and $I_{o}$ ) and the location of the forces applied to the system ( $b$ and d). In summary, the analysis of the mechanics of a hand-held rod rotating as a rigid body around the vertical axis at the wrist and striking the aperture edge reveals the force $R_{z}$ acting on the tissues along the longitudinal axis that is related to the muscular force $F$ as follows:

$$
R_{z}=-F\left\{\sin \alpha\left[1-(2 d / b)+\left(\operatorname{mad} / I_{o}\right)\right]\right\} .
$$

The quantity in the braces, called $\lambda_{e}$, is uniquely determined by the spatial configuration and the physical properties of the exploratory system. Consequently, the hypothesis has been put forth that $\lambda$, a sum of the two $\lambda_{e} s$ calculated for two aperture edges, constrains perception of the aperture size, as expressed in Equation A6:

$$
S^{\prime} \propto \lambda
$$

(Manuscript received October 17, 1997; revision accepted for publication August 13, 1998.) 